\title{
New Fresnel Köhler Optical Design: 9-Fold Photovoltaic concentrator
}

\author{
João Mendes-Lopes , Pablo Benítez , Pablo Zamora and Juan Carlos Miñano
}

\begin{abstract}
Non-uniform irradiance patterns created by Concentrated Photovoltaics (CPV) concentrators over Multi-Junction Cells (MJC) can originate significant power losses, especially when there are different spectral irradiance distributions over the different MJC junctions. This fact has an increased importance considering the recent advances in 4 and 5 junction cells. This work presents a new CPV optical design, the 9-fold Fresnel Köhler concentrator, prepared to overcome these effects at high concentrations while maintaining a large acceptance angle, paving the way for a future generation of high efficiency CPV systems of 4 and 5 junction cells.
\end{abstract}

Keywords: Nonimaging, concentrator, photovoltaic, solar energy, Köhler integration, SoG, irradiance distribution

\section{INTRODUCTION}

Concentrator Photovoltaic (CPV) systems are presented as a solution to achieve high efficiency on the conversion of Sun light into electric power, by concentrating light into a small Multi-Junction cell (MJ) using low cost optical materials. The optics systems have to ensure a good optical efficiency, large acceptance angle, and good irradiance distribution on the cell, while maintaining a high concentration $\mathrm{C}_{\mathrm{g}}$. The acceptance angle $(\alpha)$ provides tolerance against tracking misalignments and manufacturing errors, and has a tradeoff with the concentration. This tradeoff is described through the concentration-acceptance angle product $\mathrm{CAP}$, defined by $C_{\mathrm{g}}{ }^{1 / 2} \sin \alpha$, being an appropriate merit function for a concentrator ${ }^{1}$.

Irradiance distribution over the PV cell is also fundamental for high efficiency, due to the fact that non-uniform patterns can cause an increase in the effective series resistance of the cell, creating a drop in efficiency. In particular, the typical non-uniform irradiance patterns created by conventional CPV concentrators over MJ cells can originate significant power losses. Furthermore, when chromatic aberrations between irradiance distributions over the different junctions are significant, the effect on power losses increases considerably ${ }^{2-4}$.

The advances in 4 and 5 junction solar cells suggests that the spectrum balance and irradiance uniformity between different junctions will be increasingly important for future CPV systems. ${ }^{5}$. These cells are likely to be more expensive, therefore requiring higher concentration to be cost effective.

The most recent optical designs for CPV systems, namely Fresnel-Köhler (for this work, presented as FK4) technology, have proven that spectral balanced high irradiance uniformity is achievable, while maintaining a high acceptance angle, for concentrations of 850-1000 $\mathrm{x}^{6}$. However, when aiming for larger concentrations, irradiance uniformity decreases for a given architecture, as demonstrated in [4] (for SILO system, which has almost perfect uniformity at 300 $\mathrm{x}^{7}$ ).

This work presents a new CPV optical design, 9-fold Fresnel-Köhler (FK9) concentrator, prepared to overcome these effects while maintaining a large acceptance angle, paving the way for 4 and 5 junction based CPV systems at higher concentrations. 


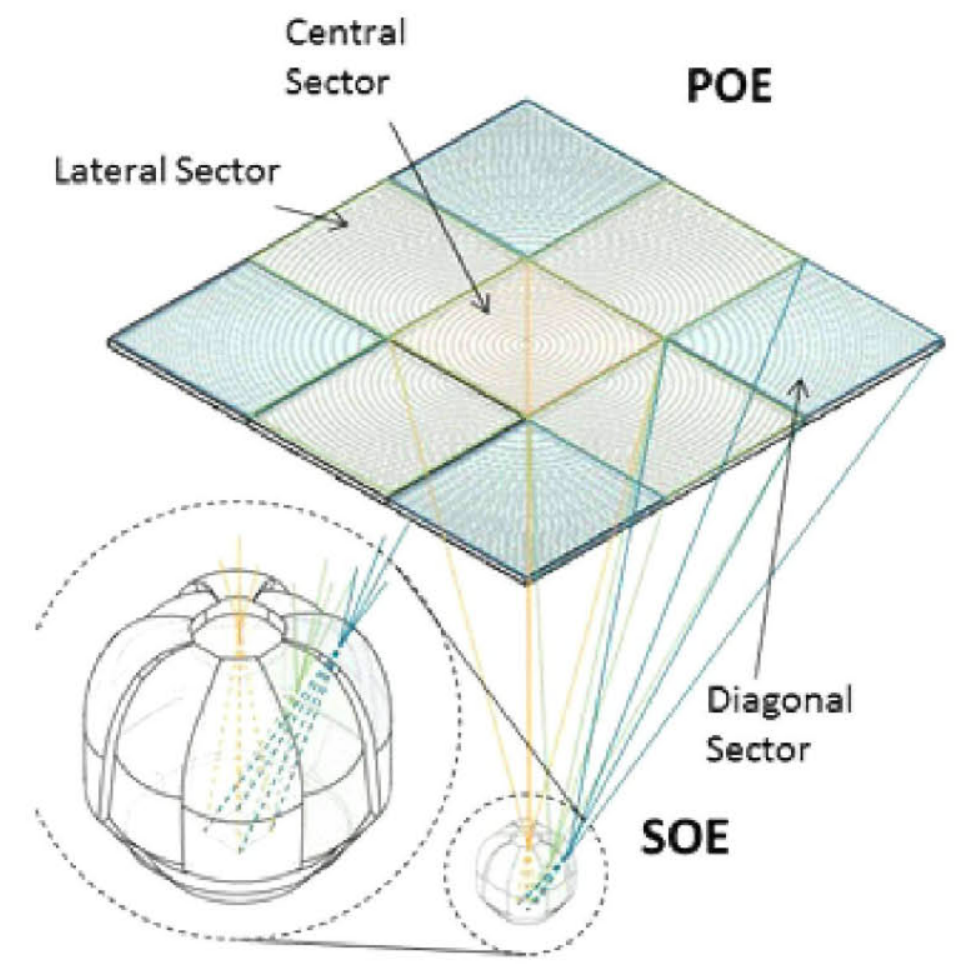

Figure 1. Schematic view of FK9 concentrator. Three different fold types, central, lateral and diagonal (in the picture, orange, green and blue, respectively) form a 9 fold system. Each sector of the SOE images the corresponding sector of the POE in the cell.

\section{TECHNICAL DESCRIPTION OF FK9}

FK9 consists on a Primary Optical Element (POE), a flat Fresnel lens divided in 9 sectors, and a Secondary Optical Element (SOE) also divided in 9 sectors, all of them free form, and coupled with the respective sector of the POE, as seen in Figure 1. The POE consists in 1 symmetric central sub Fresnel lens, four lateral and for diagonal sub Fresnel lenses, each of them symmetric with each other relative to the Fresnel lens center. The four lateral and four diagonal sublenses may be seen as off-center square pieces of a symmetric Fresnel lens.

The SOE has the same structure: 1 central sub-lens, four lateral and four diagonal sub-lenses, all symmetric with each other relative to the center axis, all of them free form. Each sub-lens is coupled with a sector of the POE, imaging each square sector of the Fresnel lens into the cell, thus performing Köhler integration. This integration allows that, even when the concentrator is misaligned with an angle within the acceptance, each surface of the SOE can still control the incoming bundle from each sector of the POE and redirecting into the entire cell surface, allowing an excellent irradiance uniformity, even for off-axis angles. The fact that the input bundle is divided into 9 different bundles enables a smaller solid angle of each bundle coming from the different sectors of the SOE. Also, allows that the thermal stress of each light spot inside the SOE is reduced by the ninth. The optical design is optimized such that the extreme rays that impinge in the POE are directed into the extreme of the cells, both for an aligned angle as for a design acceptance angle.

The integration allows an excellent spectrum and spatial irradiance on the cell, even at very high concentrations and extreme thermic conditions, as presented below. The fact that the incoming bundle of Sun rays is divided into nine channels enables a very high acceptance for rays coming from any direction, thus allowing a high CAP. Moreover, this incoming light split reduces the risk of glass solarization in the SOE if compared with other conventional Fresnel-based concentrators, where a single light focus appears inside the SOE.

FK9 optical surfaces, are very similar to those in other conventional concentrators, from a manufacture point of view. The POE of the FK9 is almost identical to conventional flat Fresnel lenses, while the SOE is equivalent to typical dome- 
shaped SOEs used in other concentrators. This implies that the same low-cost and already mature manufacturing techniques, such as compression molding, hot embossing and glass molding, can be applied to this concentrator, thus the production cost is approximately the same as for this methods. Also, the potential for production cost reduction is increased due to loose assembly/ alignment tolerances (through high acceptance angle).

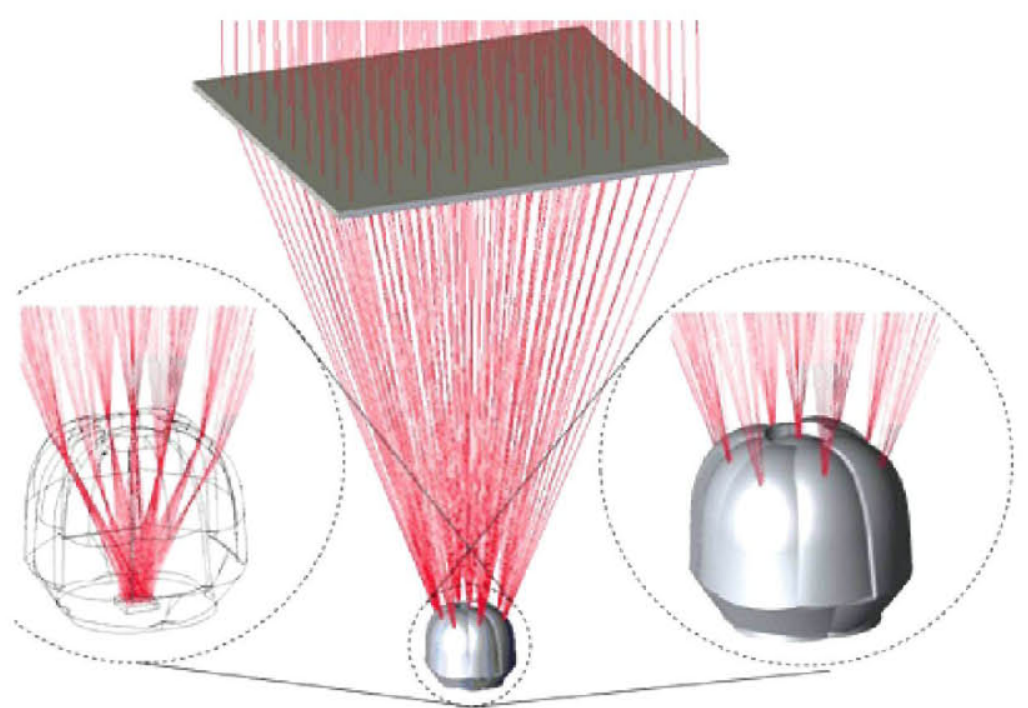

Figure 2. FK9 ray tracing simulation, where the 9 focus on SOE sectors are visible, as well as the image on the cell

\section{SIMULATION RESULTS FOR $\mathrm{C}_{\mathrm{G}}=1000 \mathrm{X}$}

Simulation results are presented for a FK9 designed for a geometrical concentration of $\mathbf{C}_{g}=\mathbf{1 0 0 0 X}$ (over cell illuminated area) and a F-number of $\mathbf{1}$. All the following simulation results correspond to raytracing simulations carried out under the same realistic conditions: AM1.5D sunlight spectrum, finite sun $\left( \pm 0.265^{\circ}\right)$ and Fresnel and absorption losses. POE is made on PMMA, and SOE is made on Schott B270 glass. Moreover, no anti reflecting coating has been applied on SOE. POE teeth rounded edges were not considered.

Figure 2 presents a ray tracing simulation of FK9 concentrator. The optical behavior at normal incidence of the system can be observed: each focus of the nine POE sectors impinging in the correspondent SOE sector surface are integrated, and equally distributed over the entire cell surface.

Table 1 summarizes the main results of FK9 optical performance, and Figure 3 presents its acceptance curve. 1000x FK9 has an acceptance angle of $\pm 1.17^{\circ}$. Also, the curve almost maintains its maximum efficiency until it reaches $1^{\circ}$ of deviation, ensuring a very high efficiency within the acceptance angle. The concentration level of $\mathrm{C}_{\mathrm{g}}=1000 \mathrm{x}$ and an acceptance angle of $\pm 1.17^{\circ}$ represents a CAP of 0.65 . The losses that can be observed after $1^{\circ}$ of deviation are mostly due to Fresnel reflection effects on the SOE surface. This implies that, if an optimized anti reflecting coating for the appropriated incidence angles is applied on the SOE optical surface, the acceptance angle and the CAP can be further increased, Moreover, a global efficiency increase of $2 \%$ can be expected if a proper anti reflecting coating is applied.

Table 1. 9 Fold Fresnel Köhler concentrator simulation results for $\mathrm{C}_{\mathrm{g}}=1000 \mathrm{x}$

\begin{tabular}{cc}
\hline $\mathbf{C}_{\mathrm{g}}=\mathbf{1 0 0 0 x}$ & Simulation results \\
\hline Optical Efficiency & $83.9 \%$ \\
Acceptance angle & $\pm 1.17^{\circ}$ \\
$\mathrm{C} A \mathrm{P}$ & 0.65 \\
$\mathrm{f}-$ number & 1 \\
\hline
\end{tabular}




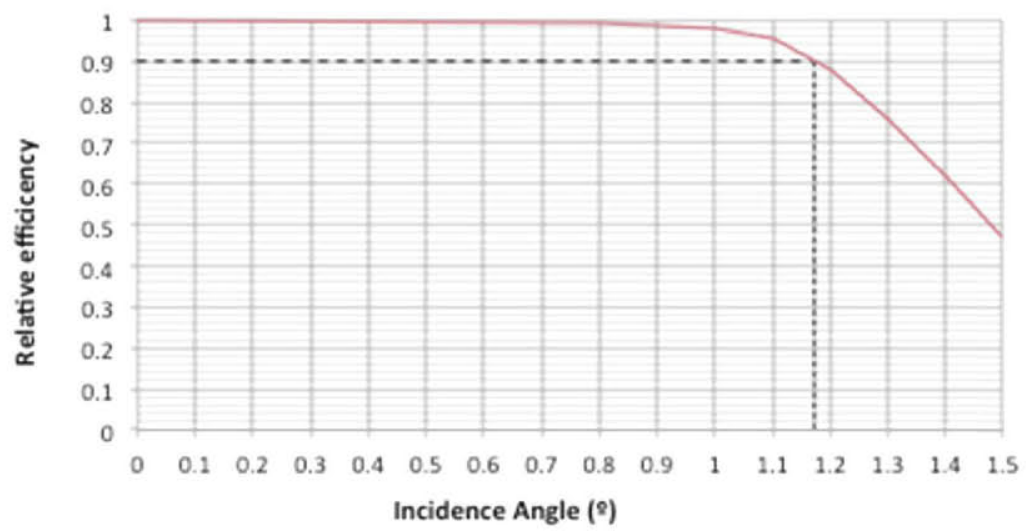

Figure 3. FK9 acceptance curve for $\mathrm{C}_{\mathrm{g}}=1000 \mathrm{x}$. The acceptance angle is $\pm 1.17^{\circ}$

Figure 4 represents the spectral irradiance distributions on each junction of a triple MJ cell that FK9 produces. Spatial irradiance is presented for two different incidence angles, at normal incidence and with a misalignment of $0.8^{\circ}$, within the acceptance angle. At normal incidence, spatial irradiance is almost perfectly uniform for all junctions. This uniform spatial irradiance is kept for the full Sun light spectrum. This is especially important to avoid current mismatches between junctions and high series resistant losses, and even more if one considers cells with 4 and 5 junctions, as they are more likely to be sensitive to spectrum variations, thus it is more difficult to achieve current mismatch.

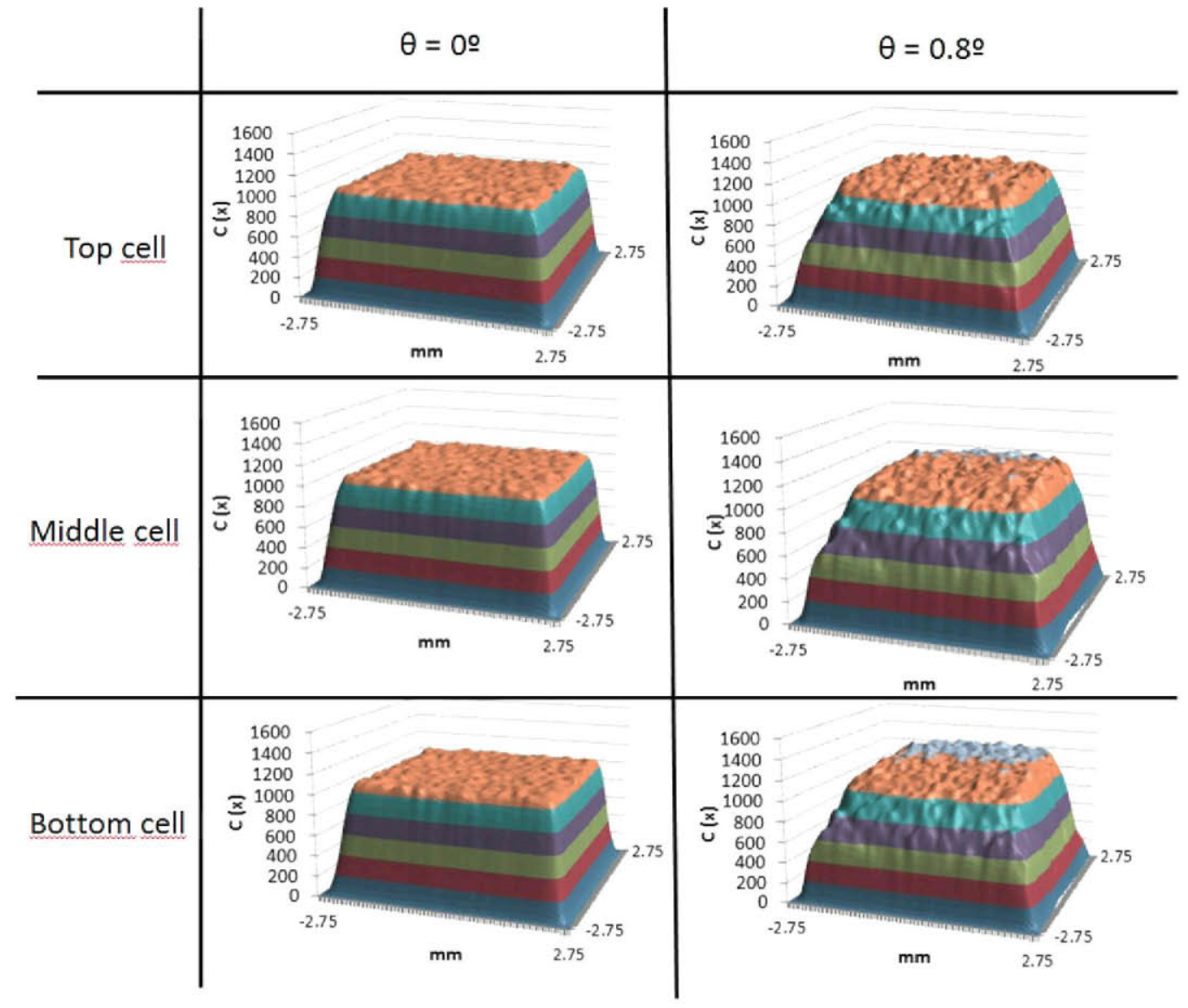

Figure 4. 1000x FK9 spectral irradiance distributions for Top, Middle, and Bottom junction of a Triple MJ cell, for normal incidence and for a misalignment angle of $0.8^{\circ}$. The irradiance distribution is analyzed throughout the full Sun light spectrum 
For a misalignment of $0.8^{\circ}$, the spatial irradiance is still very uniform, especially in the Top and Middle junctions. When dealing with the widely used Ge-bottom MJ cells, the current is typically limited by the Top and Middle junction, thus is likely that this less uniform distribution on the Bottom Cell does not have a considerable effect on the overall performance. The Köhler integration on every surface of the SOE enables that the spectral irradiance is essentially constant within the acceptance angle.

Figure 5 presents a CAP comparison between different systems, in which all of them were designed for achieving the most uniform spatial and spectral irradiance possible through Köhler integration. While FK4 and FK9 are the systems with highest performance in this field, FK9 still outperforms the FK4 concentrator, presenting a higher CAP of 0.65 and higher acceptance angle, therefore reaching a higher tolerance value.

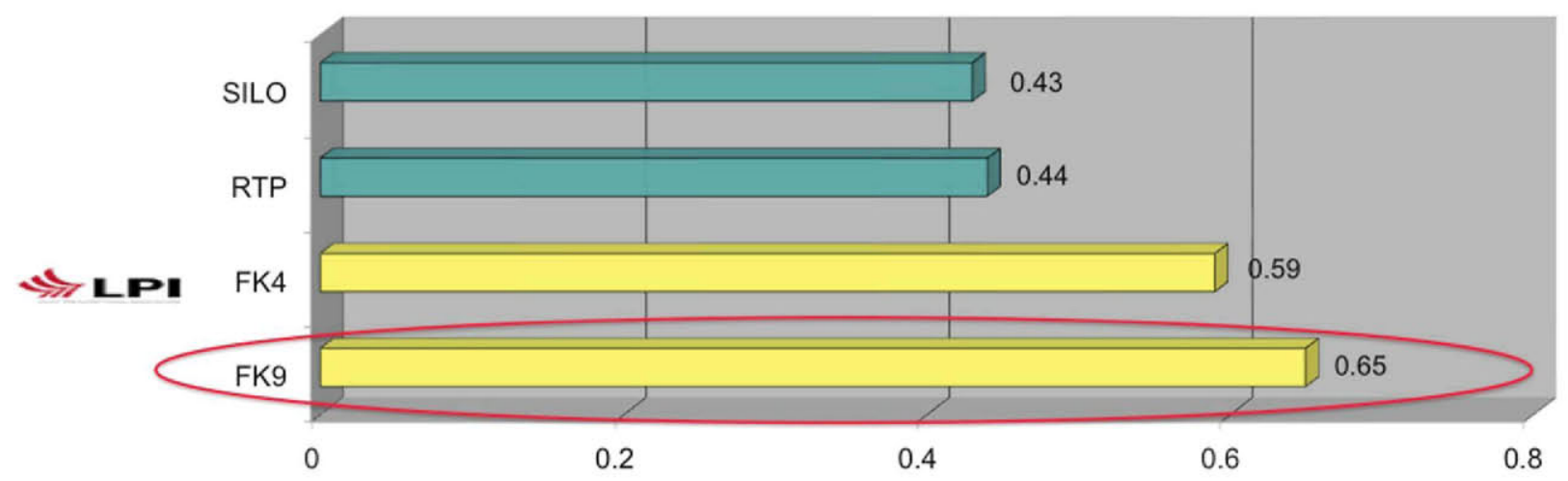

Figure 5. Comparison of CAP between different systems with rather good to excellent irradiance uniformity

\section{CONCLUSIONS}

A new CPV system, based on a 9-fold Fresnel-Köhler concentrator (FK9), was presented, with a CAP of 0.65, with $\pm 1.17^{\circ}$ for $\mathrm{C}_{\mathrm{g}}=1000 \mathrm{x}$. The system presents an excellent spatial and spectrum irradiance distribution for each junction of a Triple MJ cell.

The irradiance distribution on the different junctions of a Triple MJ cell is excellent for normal incidence, and is essentially constant within the acceptance angle, enabling that no losses due to current mismatches occur, even for misaligned angles.

FK9 paves the way for the future generation of high efficiency CPV systems of 4 and 5 junction cells.

\section{ACKNOWLEDGMENTS}

Authors thank the European Commission (SMETHODS: FP7-ICT-2009-7 Grant Agreement No. 288526, NGCPV: FP7-ENERGY.2011.1.1 Grant Agreement No. 283798), the Spanish Ministries (ENGINEERING METAMATERIALS: CSD200800066, SEM: TSI-020302-2010-65 SUPERRESOLUCION: TEC2011-24019, SIGMAMODULOS: IPT-2011-1441920000, PMEL: IPT-2011-1212-920000), and UPM (Q090935C59) for the support given to the research activity of the UPM-Optics Engineering Group, making the present work possible.

This work was partly supported by Fundação para a Ciência e Tecnologia, Scholarship SFRH / BD / 80892 / 2011.

\section{REFERENCES}

[1] R. Winston, J. C. Miñano and P. Benítez, Nonimaging Optics, New York, Elsevier-Academic Press, 2005 
[2] I. García et al, "Study of non-uniform light profiles on high concentration III-V solar cells using quasi-3D distributed models", Proceeding $33^{\text {rd }}$ IEEE Photovoltaic Specialist Conference, (2008)

[3] M. Victoria et al, "Characterization of the spatial distribution of irradiance and spectrum in concentrating photovoltaic systems and their effect on multi-junction solar cells", Prog. Photovolt: Res. Appl. doi: 10.1002/pip.1183, (2011)

[4] P. Espinet-González et al, "Triple-Junction Solar Cell Performance Under Fresnel-based Concentrators Taking Into Account Chromatic Aberration And Off-Axis Operation", $8^{\text {th }}$ International Conference on concentrating Photovoltaic systems:CPV-8, AIP Conf. Proc. 1477, pp. 81-84, Toledo, (2012)

[5] R.R. King et al, "Solar cell generations over 40\% efficiency", Prog. Photovolt: Res. Appl.. doi: 10.1002/pip. 1255, (2012)

[6] P. Zamora, et. al. "Experimental characterization of Fresnel-Köhler concentrators," J. Photon. Energy. 2(1), 021806 (2012)

[7] L.W. James, Contractor Report SAND89-7029, (1989) 